\title{
Auswirkungen von „Qualitätsoffensive“ und Honorararztwesen im Krankenhaus
}

\author{
Consequences of "Quality Offensives" and the Services of Free-Based Physicians in Hospitals
}

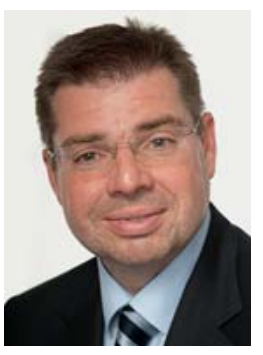

D. C. Wirtz

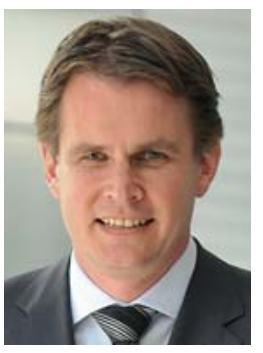

U. Stöckle

\section{Bibliografie}

DOI http://dx.doi.org/

$10.1055 / \mathrm{s}-0033-1360378$

Z Orthop Unfall 2014; 152: 18-

19 (c) Georg Thieme Verlag KG Stuttgart · New York .

ISSN 1864-6697

\section{Korrespondenzadressen Univ.-Prof. Dr. med. Dieter C. Wirtz \\ Direktor der Klinik und Poliklinik für Orthopädie und Unfallchirurgie Universitätsklinikum Bonn Sigmund-Freud-Straße 25 53105 Bonn \\ Tel.: 0228/287-14170 Fax: 0228/287-14175 \\ dieter.wirtz@ukb.uni-bonn.de}

Univ.-Prof. Dr. Ulrich Stöckle Ärztlicher Direktor BGU Klinik Tübingen Schnarrenbergstraße 96 72076 Tübingen

Tel.: 0 07071/606-1001

Fax: 07071/606-1002

ustoeckle@bgu-tuebingen.de

\section{Sehr geehrte Leserinnen und Leser, $\nabla$}

als Herausgeber unserer Zeitschrift wollen wir nicht nur zu wissenschaftlichen Fragen Stellung beziehen, sondern auch bei gegebenem Anlass uns zu entsprechenden berufspolitischen Themen äußern. Die neue Bundesregierung ist Ende 2013 mit der getroffenen Koalitionsvereinbarung für die anstehende Legislaturperiode angetreten, eine neue „Qualitätsoffensive in den Krankenhäusern“ durchzuführen. Damit rücken kosteneffiziente Leistungsgestaltung und Abteilungsführung unter dem Primat der evidenzbasierten Ergebnisqualität weiterhin in den Fokus der gesundheitspolitischen Überlegung. Dies ist grundsätzlich gut und wird zur Bündelung vorhaltekostenintensiver Bereiche auch standortübergreifend führen. Zunehmende Zentrumsbildung in Ballungsräumen mit hoher Subspezialisierung einerseits, aber auch eine notwendige „breite“ Grundversorgung in eher ländlichen Regionen andererseits sind die sich daraus ergebenden Anforderungen. Auch für unser Fach „Orthopädie und Unfallchirurgie“" werden sowohl die Kostenträger als auch die Planungsbehörden künftig mehr als vielleicht bisher darauf achten, dass Abteilungsstrukturen bedarfsgerecht an die jeweils „Vor Ort“ gegebene Situation ausgerichtet sind. Waren noch vor 5 bis 6 Jahren im Krankenhaus die Abteilungsstrukturen zur Erfüllung des Versorgungsauftrages praktisch ausschließlich durch das klassische Struktursystem „Chefarzt - Oberarzt - Assistenzarzt“ geprägt, so hat sich zwischenzeitlich doch in vielen Kliniken - und zwar auch politisch gewollt - eine „neue Säule“ durch Einbindung von Honorarärzten etabliert.

Durch das „Vertragsarztrechtsänderungsgesetz“ (VÄndG) wurde die bis dahin rechtliche Bewertung (BSG-Urteil 28.02.2007), dass Kernleistungen (wie z.B. Operationen) im Krankenhaus nur durch angestellte Ärzte des Krankenhausträgers zu erbringen sind, aufgehoben. Nach $\S 20$ Abs. 2 VÄndG ist „die Tätigkeit in oder die Zusammenarbeit mit einem zugelassenen Krankenhaus .... mit der Tätigkeit des Vertragsarztes vereinbar“. Mit dem nachfolgenden Versorgungsstrukturgesetz [01.01.2012] wurde die rechtliche Auslegung noch weiter für das ambulante Operieren im Krankenhaus präzisiert, sodass dieses durch niedergelassene, freiberuflich tätige Ärzte ausdrücklich zulässig ist. Darüber hinaus wurde mit Änderung des Krankenhausentgeltgesetzes [KHentgeltG 01.01.2013] auch die Erbringung von vollund teilstationären Leistungen durch Vertrags-/
Honorarärzte legalisiert und als abrechenbar definiert. Nach § 17 Abs. 3 KHentgeltG dürfen wahlärztliche Leistungen aber nur von angestellten oder beamteten Ärzten des Krankenhauses erbracht werden. Nicht angestellte Honorarärzte sind somit nicht liquidationsberechtigt.

Was bedeutet nun in diesem Kontext „Honorararzt"? Ein wirklich einheitliche Verwendung des Begriffes gibt es nicht. Nach Definition der Bundesärztekammer (2011) sind Honorarärzte „Fachärzte/-innen, die in medizinischen Einrichtungen zeitlich befristet freiberuflich auf Honorarbasis tätig sind“. Dabei haben sich in der klinischen Praxis vornehmlich zwei „Formen“ des Honorarztes mit ganz unterschiedlicher rechtlicher Stellung herauskristallisiert: (1) Der freiberuflich tätige Arzt, der selbstständig und eigenverantwortlich gegen Honorar arbeitet und keine Arbeitnehmerschutzrechte hat; (2) der selbstständige Arzt, der im Krankenhaus mit einem Teilzeitarbeitsvertrag angestellt und damit sozialversicherungspflichtig ist, und sowohl dem Direktionsrecht des Arbeitgebers als auch dem chefärztlichen Weisungsrecht unterliegt.

Beide „Varianten“ des Honorararztes mögen aus Sicht der Krankenhausträger durchaus attraktiv sein. Beide können zur Kompensation vom „Ausfallzeiten“ und „Auslastungsspitzen“ herangezogen werden. Dies gilt insbesondere in Abteilungen, wo Ärztemangel und Arbeitszeitgesetz zur Minderauslastung der gegebenen Ressourcen führen („keine OPs nach 16:00“). Eine spezifische Erweiterung des Leistungsspektrums (z.B. durch die Handchirurgie), welche über das bisherige „Angebot“ der Fachabteilung hinausgeht, kann ebenso zur Erlösoptimierung als auch zum Schließen einer Versorgungslücke beitragen.

Dabei spart der Krankenhausträger beim „Honorararzt ohne Teilzeitarbeitsvertrag“ zusätzlich noch die Sozialversicherungsbeiträge ein und muss sich aus rechtlicher Sicht bei der Vergütungshöhe nicht an den Vergleichsmaßstab der tarifvertraglich geregelten Lohnzahlung festangestellter Krankenhausärzte gebunden fühlen. Der umsichtige Krankenhausträger sollte hier aber nicht außer Acht lassen, dass durch ein Ungleichgewicht in der Gehaltsstruktur zu den festangestellten Ärzten Unzufriedenheit und Störungen des Betriebsfriedens vorprogrammiert sind. Als weiteres Negativum muss sich der Krankenhausträger auch bewusst sein, dass er durch „honorarärztliche Versorgungskonstrukte ohne Anstellungsvertrag“ sich dem Vorwurf der „verdeckten Kopfpauschale“ aussetzt und nicht nur die 
Leistungserbringung, sondern auch die Abrechnung in der „Wahlarztkette“ nicht gegeben sind. Insofern kann den Krankenhausträgern nur angeraten sein, sofern die Betätigung von Honorarärzten zur bedarfsgerechten Deckung des Versorgungsauftrages notwendig ist, ausschließlich „Honorarärzte mit Teilzeitarbeitsvertrag“ anzustellen.

Dabei muss es in Absprache und Übereinstimmung mit dem Chefarzt der Fachabteilung eine klare und eindeutige Regelung hinsichtlich Zuständigkeiten, Haftung und Einbindung in die Klinikhierarchie geben. Auch der „Honorararzt mit Teilzeitarbeitsvertrag“ muss in die Strukturqualität und Prozessoptimierung der Klinik eng eingebunden sein. Er muss sich in die Weiterbildung der jüngeren Kollegen einbringen und Teamverantwortung übernehmen. Dies gelingt nur, wenn auch der „Honorararzt mit Teilzeitarbeitsvertrag“ sich kontinuierlich und langfristig engagiert. Bettenführende Abteilungen brauchen Kontinuität, kurzfristige Arztwechsel stören die Arzt-Patienten-Beziehung. Qualitätsüberprüfungen im Rahmen von Zertifizierungsprozessen sollten daher künftig nicht nur die jeweilige Institution bewerten, sondern auch individualisiert die handelnden Ärzte. Damit müssen sich auch Honorarärzte im Krankenhaus der externen Bewertung unterziehen. Nur dann kann die „Marke“ Krankenhaus nachhaltig medizinischen und wirtschaftlichen Erfolg verbuchen.

So attraktiv das Honorararztwesen für den Vertragsarzt einerseits als auch für den ein oder anderen Krankenhausträger unter den gegebenen rechtlichen Rahmenbedingungen auch sein mag, eine Fachabteilung im Krankenhaus braucht einen Chefarzt oder ein Chefarztteam. Dieses ist und bleibt - gerade wegen der politisch initiierten und geforderten Qualitätsoffensive im Krankenhaus - das Modell der Zukunft. Ohne einen Gesamtverantwortlichen als Leistungsträger und Vorbild, der auch das Krankenhaus mit prägt, ist eine bedarfs- und ergebnisorientierte Patientenversorgung nicht möglich.

\section{D.C. Wirtz, U.Stöckle}

